\title{
Effects of frontal lobe damage on interference effects in working memory
}

\author{
SHARON L. THOMPSON-SCHILL \\ University of Pennsylvania, Philadelphia, Pennsylvania \\ JOHN JONIDES \\ University of Michigan, Ann Arbor, Michigan \\ CHRISTY MARSHUETZ \\ Yale University, New Haven, Connecticut \\ EDWARD E. SMITH \\ University of Michigan, Ann Arbor, Michigan \\ MARK D'ESPOSITO \\ University of California, Berkeley, Califormia \\ IRENE P. KAN \\ University of Pennsylvania, Philadelphia, Pennsylvania \\ ROBERT T. KNIGHT \\ University of California, Berkeley, California \\ and \\ DIANE SWICK \\ University of California, Davis, California
}

\begin{abstract}
Working memory is hypothesized to comprise a collection of distinct components or processes, each of which may have a unique neural substrate. Recent neuroimaging studies have isolated a region of the left inferior frontal gyrus that appears to be related specifically to one such component: resolving interference from previous items in working memory. In the present study, we examined working memory in patients with unilateral frontal lobe lesions by using a modified version of an item recognition task in which interference from previous trials was manipulated. In particular, we focused on patient R.C., whose lesion uniquely impinged on the region identified in the neuroimaging studies of interference effects. We measured baseline working memory performance and interference effects in R.C. and other frontal patients and in age-matched control subjects and young control subjects. Comparisons of each of these groups supported the following conclusions. Normal aging is associated with changes to both working memory and interference effects. Patients with frontal damage exhibited further declines in working memory but normal interference effects, with the exception of R.C., who exhibited a pronounced interference effect on both response time and accuracy. We propose that the left inferior frontal gyrus subserves a general, nonmnemonic function of selecting relevant information in the face of competing alternatives and that this function may be required by some working memory tasks.
\end{abstract}

This research was supported by NIH Grant MH60414, NSF Grant SES9973391, the Searle Scholars Program, and the University of Pennsylvania Research Foundation. A preliminary version of this study was reported at the annual meeting of the Society for Neuroscience in 1999. We thank Jeris Minor, Mike Herzlinger, and Jessica Panzer for their assistance in coding data and testing control subjects, Clay Clayworth for preparing the lesion figures, Donatella Scabini and Marianna Stark for identifying and scheduling patients, Brad Postle for help in assembling stimuli and for general advice, Saul Sternberg, Mark Jung-Beeman, and three anonymous reviewers for helpful comments on an earlier version of this manuscript, and all of our patients for the generous contribution of their time and effort. Correspondence regarding this manuscript should be addressed to S. L. Thompson-Schill, Department of Psychology, University of Pennsylvania, 3815 Walnut Street, Philadelphia, PA 19104-6196 (email: thompson@ psych.upenn.edu).
The ability to temporarily store and manipulate information is necessary in many cognitive domains. Since the advent of cognitive psychology, theories about the processes involved in temporarily holding information in a limited capacity memory system have continued to evolve. Early theories of a unitary short-term memory store that prevailed in the 1960s (e.g., Atkinson \& Shiffrin, 1968) were eventually replaced with theories of a multicomponentialmemory system dubbed working memory. In a very influential theory of working memory, three distinct components of the working memory system are described: an articulatory loop used to rehearse verbal material, a visuospatial sketch- 
pad used to rehearse nonverbal images, and a central executive that controls these rehearsal processes (Baddeley, 1986). Research over the past two decades has supported the theory that working memory is not a unitary system.

Strong evidence for a multicomponent working memory system comes from cognitive neuroscience investigations that have identified brain areas that appear to be selectively involved in only one of the components of working memory. For example, some patients have impaired working memory for verbal information (Basso, Spinnler, Vallar, $\&$ Zanobio, 1982), whereas other patients have impaired working memory for spatial information (Hanley, Young, $\&$ Pearson, 1991). Functional neuroimaging studies have supported the idea that the verbal working memory system can be further decomposed into two distinct systems: one that temporarily maintains phonological information and another that actively rehearses that information. For example, Awh et al. (1996) found PET activation attributable to the phonological buffer in the left parietal cortex and activation attributable to verbal rehearsal in the left prefrontal cortex.

In recent years, frontal lobe function has become almost synonymous with working memory. Evidence that the frontal lobes play an important role in working memory goes back more than half a century, to early reports that frontal lobe damage in nonhuman primates causes an inability to remember the location of hidden food after a brief delay (e.g., Jacobsen, 1935). More recent eletrophysiological recording studies in monkeys have further strengthened this link by demonstrating that some neurons in the frontal lobes fire only during the delay period of a working memory task (Fuster \& Alexander, 1971). Furthermore, some of these neurons fire during spatial working memory tasks, whereas others fire during nonspatial working memory tasks, consistent with the idea of a multicomponential nature of working memory (Wilson, Scalaidhe, \& GoldmanRakic, 1993).

Despite many sources of evidence that the frontal lobes are involved in working memory, investigations of the effects of frontal lobe damage on working memory have been somewhat equivocal. Several laboratories have reported no effect of frontal lobe damage on working memory performance (e.g., Baldo, 1997; Ghent, Mishkin, \& Teuber, 1962). Others have reported working memory impairments following frontal lobe damage (e.g., Chao \& Knight, 1998). Still other groups have reported working memory deficits in some patients with frontal lesions, but not in others, or under some conditions, but not under others (e.g., Bechara, Damassio, Tranel, \& Anderson, 1998; Ptito, Crane, Leonard, Amsel, \& Caramanos, 1995). In a recent review of neuropsychological literature on working memory, D'Esposito and Postle (1999) concluded that frontal lobe lesions may impair certain components of working memory that are not present in simple span tasks (on which patients with frontal lesions are unimpaired) and that are present in some delayed response tasks, but not in others. For example, delayed response tasks that included a distraction task during the delay were more likely to reveal im- pairments in patients with frontal lobe lesions. D'Esposito and Postle conjectured that the frontal lobes may support a nonmnemonic component (or components) of working memory.

Jonides and colleagues used functional neuroimaging to test the hypothesis that the prefrontal cortex mediates nonmenomic functions in working memory (Jonides, Smith, Marshuetz, Koeppe, \& Reuter-Lorenz, 1998). In particular, they measured the extent to which activity in the prefrontal cortex was sensitive to interference across trials. Subjects were shown a group of letters on each trial, followed by a brief delay. At the end of the trial, the subjects had to decide whether a probe letter was a member of the target set of letters shown on that trial. The critical manipulation in this experiment was whether the probe item was a member of the target set on a previous trial. Behavioral data indicated that the subjects were slower and less accurate at rejecting a negative probe item if that item was a member of a recently presented trial. In a comparison of blocks of trials having many such items with blocks of trials in which probe items were never recently presented, PET data revealed increased activity in ventrolateral aspects of the left prefrontal cortex (Brodmann's area 45; see Figure 1). This result was confirmed in an event-related fMRI study that permitted specification of this effect to the response period (D'Esposito, Postle, Jonides, \& Smith, 1999).

Owing to the correlational nature of functional neuroimaging methods, inferences about the necessity of particular brain regions for cognitive functions cannot be made on the basis of results from PET or fMRI studies. In contrast, examination of specific deficits resulting from focal brain injury affords one the ability to draw causal inferences about structure-function relations. Thus, neuropsychological investigations of cognitive deficits provide a natural complement to neuroimaging studies. In the present study, we investigated the necessity of the prefrontal cortex for working memory performance. Specifically, we assessed interference effects in working memory, as revealed by the above task, in patients with damage to regions of the left and right prefrontal cortex.

\section{METHOD}

\section{Subjects}

Seven patients with unilateral damage to the lateral prefrontal cortex were identified on the basis of a focal frontal cortex lesion evident on computerized tomography (CT) or magnetic resonance imaging (MRI) scans. All the patients were recruited from the Outpatient Clinic of the VA Northern California Health Care System and other local Northern California hospitals. Lesions were identified on MRI or CT scans obtained at a chronic stage, were transcribed onto corresponding axial templates by two independent raters, and were projected onto a lateral view of the brain (see Figures 2 and 3). None of the subjects had a history of psychiatric problems or substance abuse. All the subjects were paid for their participation and gave their informed consent.

One patient, R.C., was of critical importance for the present investigation, owing to the unique location of his lesion (see Figure 2). At the time of testing, R.C. was a 51-year-old male with a significant lesion $(53 \mathrm{cc})$ in the anterior aspects of the left middle and in- 


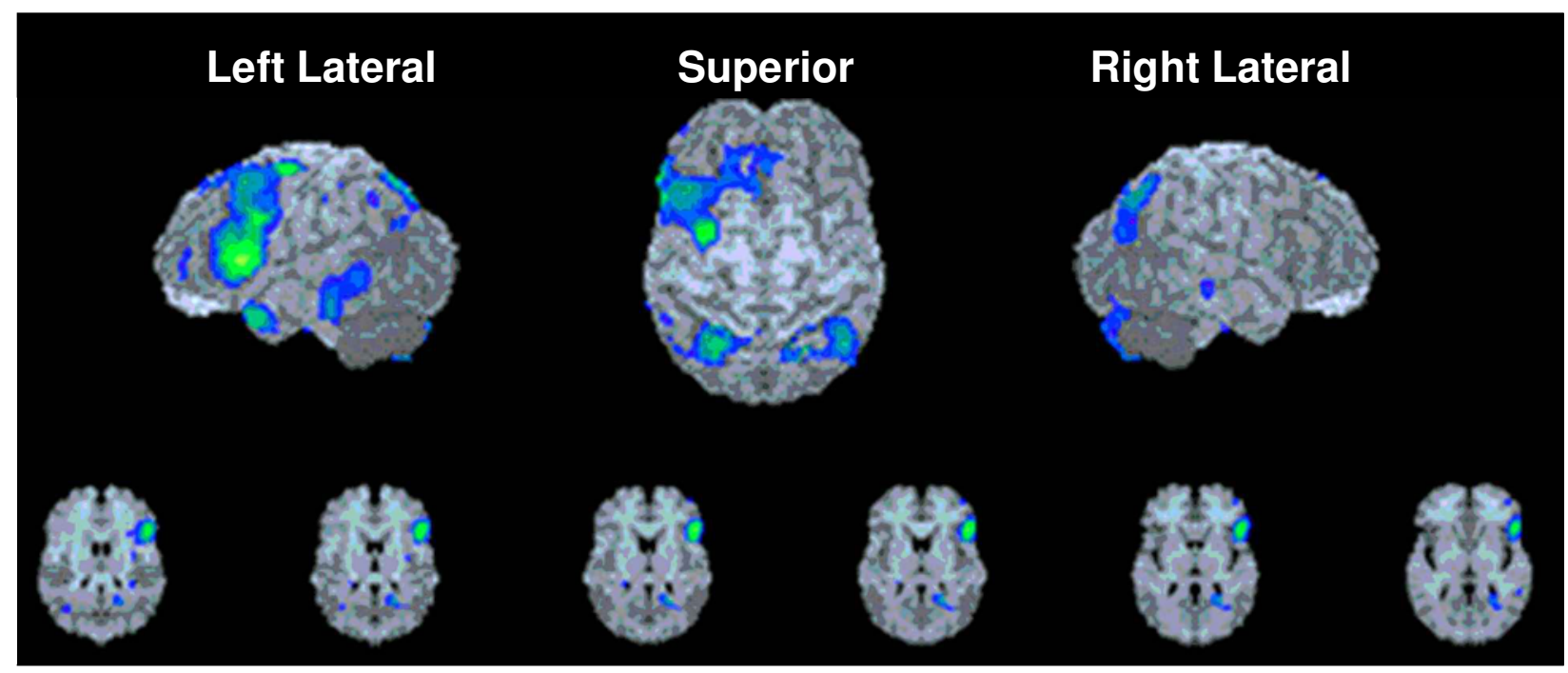

Figure 1. PET activation associated with item recognition on trials with recently presented probe items, as compared with trials with nonrecently presented probe items. Activation in the lateral prefrontal cortex was centered in the region corresponding to Brodmann's area 45 of the left inferior frontal gyrus. From "Inhibition in Verbal Working Memory Revealed by Brain Activation," by J. Jonides, E. E. Smith, C. Marshuetz, R. A. Koeppe, and P. A. Reuter-Lorenz, 1998,Proceedings of the NationalAcademy of Sciences, 95 , p. 8411. Copyright 1998 by the National Academy of Sciences. Adapted with permission.

ferior frontal gyri. His lesion was the result of a ruptured arteriovenous malformation of the left middle cerebral artery with a subarachnoid hemorrhage, followed by surgery, nearly 20 years prior to testing. The location of R.C.'s lesion, identified from an MRI scan obtained 1 year prior to testing, was relevant for the present investigation because it had an extent and location remarkably similar to those for the activation related to interference on a working memory test, described above (Jonides et al., 1998).

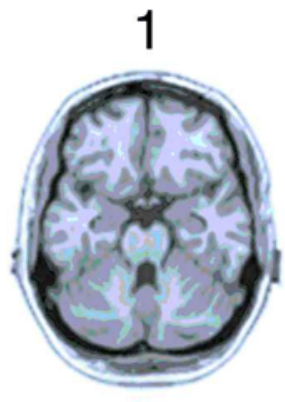

$-18$

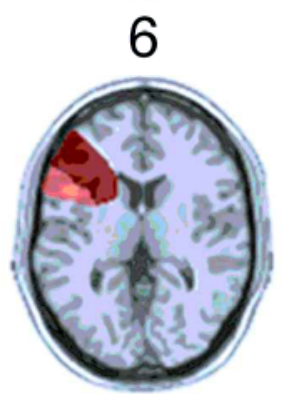

$+12$

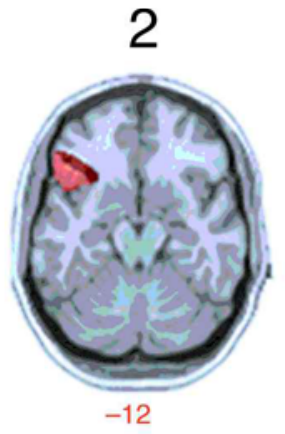

7

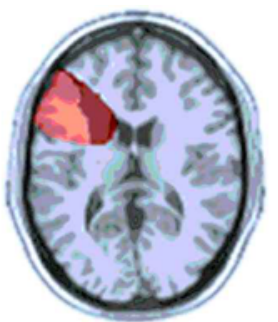

$+18$

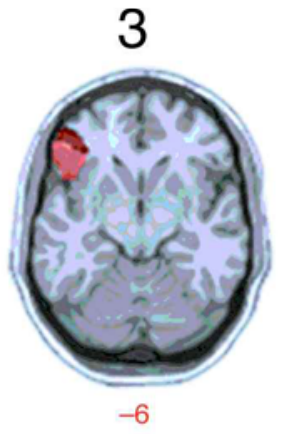

8

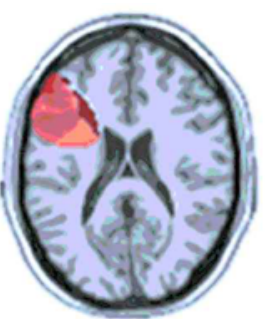

$+24$

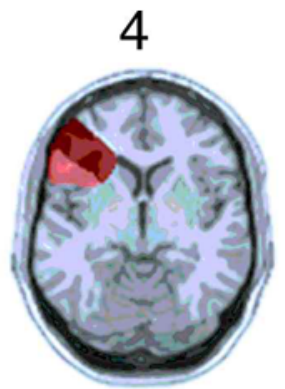

0

9

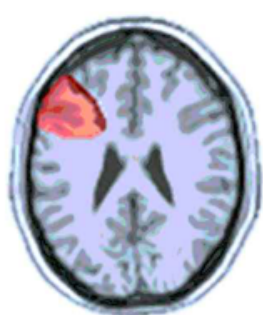

$+30$

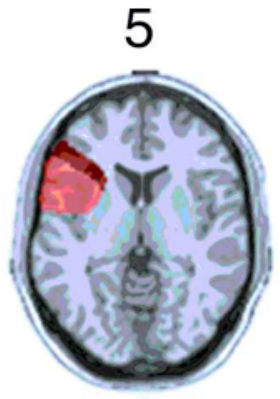

$+6$

10

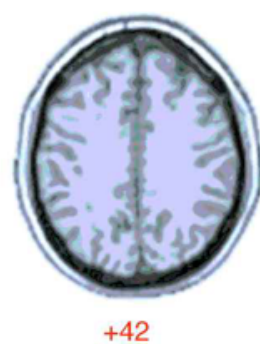

Figure 2. Reconstruction of patient R.C.'s lesion. His lesion is indicated by red shading overlayed on a reference MRI image; the $z$ coordinates are indicated below each slice $(z=-18$ to +42$)$. The dark red shading indicates the portion of R.C.'s lesion that was unique in comparison with the frontal control subjects. The light red shading indicates the portion of R.C.'s lesion that was shared by at least 1 other patient in this study. Patient R.C. was selected because of the correspondence between his lesion and the locus of activity in the neuroimaging study of interference (compare with Figure 1). Damage indicated on Slices 3-6 indicates the specific region of interest. 
W.E.

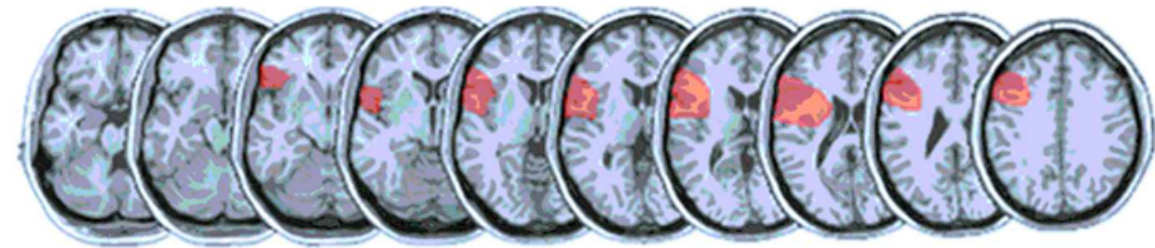

M.F.

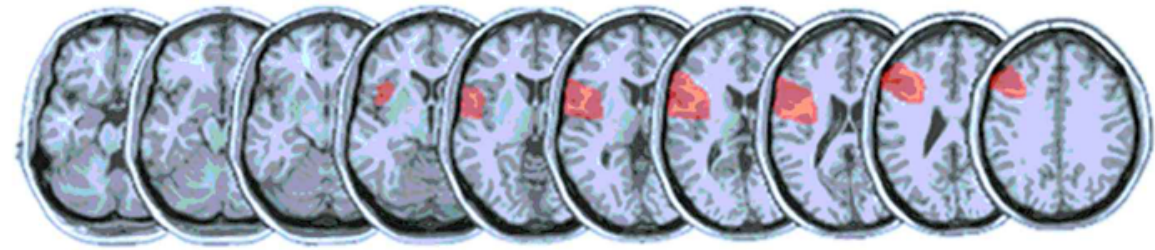

J.M.

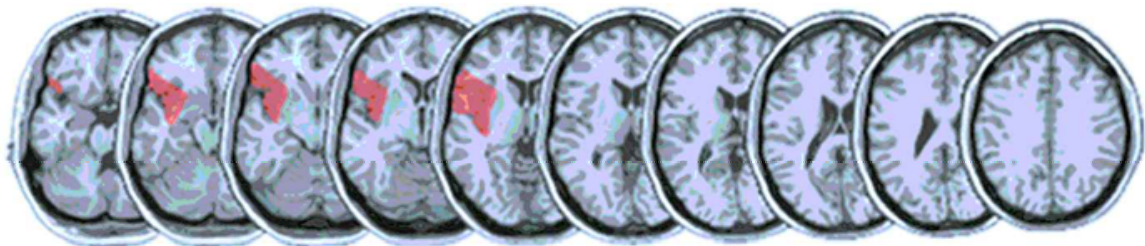

W.A.

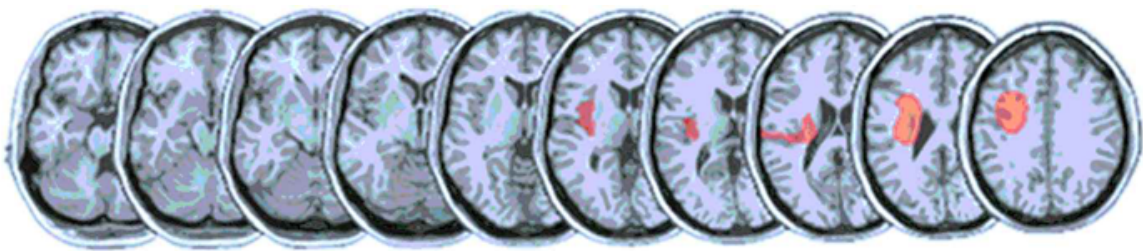

N.T.

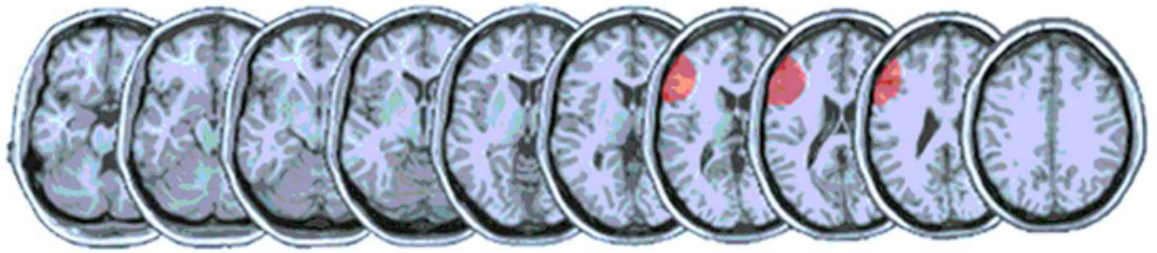

E.B.
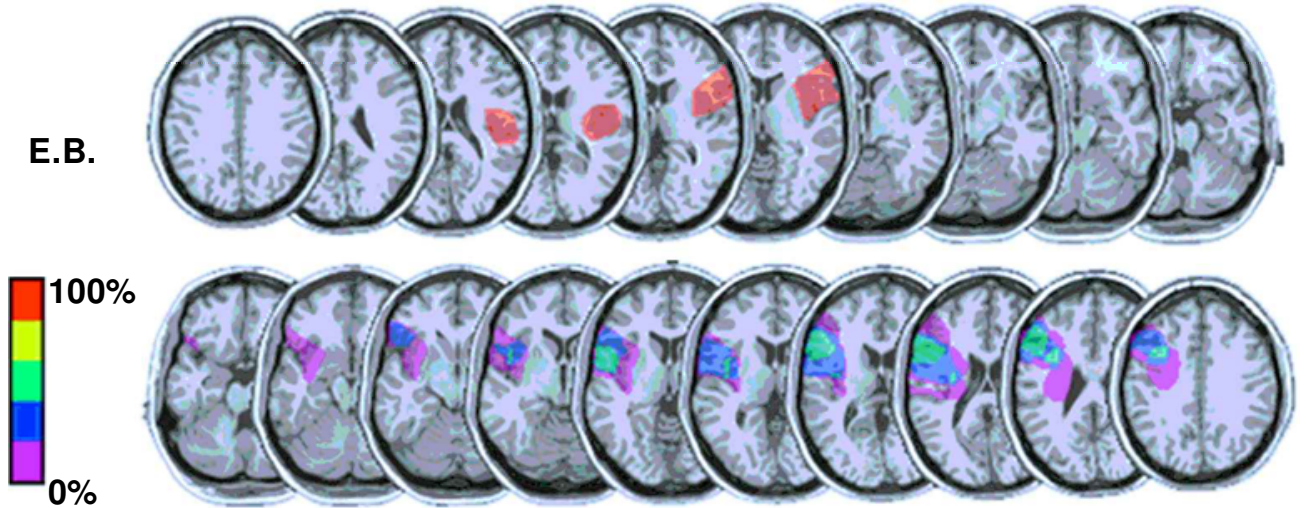

Figure 3. Reconstructions of lesions in 6 frontal control subjects based on computerized tomography and magnetic resonance scans. Ten slices are shown for each subject $(z=-18$ to +42$)$, corresponding to the 10 slices displaced for R.C. in Figure 2. Note that Patient E.B.'s images are in the reverse order to allow display of her right-hemisphere lesion. The bottom row displays the lesions for 5 patients (excluding E.B.) superimposed to illustrate the common lesion locations in the control group.

The remaining 6 patients ( 3 males, 3 females) had lesions to more posterior or superior aspects of the lateral prefrontal cortex in either the right or the left hemisphere $(M=27.2 \mathrm{cc})$ as the result of a single infarction of the precentral branch of the middle cerebral artery (see Figure 3). The average age of these patients was 67.7 years (ranging from 56 to 81 years).

Three control groups were established for comparison to the patients with frontal lesions. All three groups consisted of healthy volunteers 
Table 1

Two Consecutive Sample Trials for Each of the Trial Types in the Item Recognition Task

\begin{tabular}{|c|c|c|c|c|}
\hline \multirow[b]{2}{*}{ Condition (Trial $n$ ) } & \multicolumn{2}{|c|}{ Trial $n-1$} & \multicolumn{2}{|c|}{ Trial $n$} \\
\hline & Target Set & Probe & Target Set & Probe \\
\hline Control positive & $\mathrm{gkpn}$ & $\mathrm{D}$ & $\mathrm{sfhm}$ & $\mathrm{F}$ \\
\hline Control negative & w b t q & $X$ & $\mathrm{czrv}$ & $\mathrm{J}$ \\
\hline Nonrecent positive & $\mathrm{m} \mathrm{d} \mathrm{s} \mathrm{k}$ & M & $\mathrm{sfkt}$ & $\mathrm{T}$ \\
\hline Nonrecent negative & $\mathrm{vnbf}$ & $\mathrm{C}$ & $\mathrm{rbjn}$ & $\mathrm{D}$ \\
\hline Recent positive & $\mathrm{krzb}$ & $\mathrm{N}$ & $\mathrm{btks}$ & $\mathrm{B}$ \\
\hline Recent negative & hlwp & $\mathrm{L}$ & $\mathrm{kpwn}$ & $\mathrm{H}$ \\
\hline
\end{tabular}

Note-The trial type indicated in each row corresponds to the second trial in the table (trial $n$ ).

with no history of neurological disease, substance abuse, or psychiatric disorders. The first group was matched in age and education to patient R.C. This group of 8 subjects ( 4 males, 4 females) had a mean age of 51.5 years (ranging from 45 to 60 ). The second group was matched in age and education to the remaining 6 frontal patients. This group of 6 subjects ( 3 males, 3 females) had a mean age of 62.3 years (ranging from 54 to 81 ) that was comparable to the group of frontal patients $(t<1)$. Finally, a group of 32 young subjects (mean age of 18.7 years) was tested for purposes of comparison with all of the above patient and control groups.

\section{Procedure}

The design of the experiment was a modification of the experimental paradigm used in the neuroimaging studies of Jonides et al. (1998) and D'Esposito et al. (1999). Each subject completed 160 trials of an item recognition memory task, in which they were required to judge whether a test probe item was a member of a set of studied items. At the beginning of each trial, a "Get Ready" cue was presented for $1,000 \mathrm{msec}$. This was followed by a cross in the center of the screen, on which the subjects were instructed to fixate. After $500 \mathrm{msec}$, the target set was presented. The target set was a visual display of four lowercase letters arranged above, below, to the left, and to the right of a central fixation cross. The target set remained on the screen for $1,500 \mathrm{msec}$, followed by a 3,000-msec delay. Following this delay, the probe (i.e., a single uppercase letter) appeared in the central location, and the subject was instructed to indicate whether that probe was a member of the current target set or not.

The subject indicated his or her responses by pressing a key labeled either yes or no with one finger of his or her dominant hand (except in cases of hemiplegia of the dominant hand). The response device had three buttons. Throughout the trial, the subject rested a finger on the central button. To respond, the subject had to move the finger either to the left or to the right to press the correct response button. There was a 5,500-msec time limit for the response, but the subjects were instructed to respond as soon as they knew the answer. The subjects were also instructed to guess if they were not certain. No feedback was given. Trials were grouped into four 40-trial blocks that were separated by approximately $1 \mathrm{~min}$. Prior to the experimental trials, the subjects completed a 16-trial practice block; several patients required a second practice block to ensure understanding of the task.

There were four types of trials arranged pseudorandomly throughout the experiment. Half of the trials contained probe items that were members of the current target set (positive trials), and half of the trials contained probe items that were not members of the current target set (negative trials); if responding correctly, the subjects would give a yes response to the positive trials and a no response to the negative trials. For both positive and negative trials, half of the trials contained probe items that were members of the previous target set (recent trials), and half of the trials contained probe items that were not members of either of the previous two target sets (nonrecent trials). For all the trials, two of the four letters in the target set were repeated from the previous trial, so that repetition of items in the target set was not confounded with trial type. The location of items in the target array was randomly determined, so a repeated item had a .25 chance of occurring in the same location on two consecutive trials. The recent- negative trials were the trials that were associated with increased activity in the left inferior frontal gyrus in neuroimaging studies (D'Esposito et al., 1999) and, therefore, were the trials that were critical for the present investigation.

Following this procedure, each subject completed one 40-trial block of a control task. The control trials were nearly identical to those described above, although there was no repetition of target or probe items between trials. Specifically, the target letters used on each trial were not present (as targets or probes) in either of the two previous trials. This control task served as a baseline measure of working memory ability, in the event that item repetition on both the recent and the nonrecent trials would produce performance impairments. Examples of each of the trial types are given in Table 1 .

\section{RESULTS}

For each of the six trial types (control-positive, controlnegative, nonrecent-positive, nonrecent-negative, recentpositive, and recent-negative), accuracy and median response time (to correct items) were calculated for each subject; mean error rate and mean median response time for

Table 2

Mean Median Response Latencies (in Milliseconds; With Standard Deviations) for All Subject Groups for Six Trial Types of the Item Recognition Task

\begin{tabular}{|c|c|c|c|c|c|c|c|c|c|c|c|c|c|c|}
\hline \multirow{4}{*}{$\begin{array}{l}\text { Subject } \\
\text { Group }\end{array}$} & \multicolumn{12}{|c|}{ Trial Type } & \multirow{3}{*}{\multicolumn{2}{|c|}{$\begin{array}{l}\text { Interference } \\
\quad \text { Effect } \\
\end{array}$}} \\
\hline & \multicolumn{4}{|c|}{ Control } & \multicolumn{4}{|c|}{ Nonrecent } & \multicolumn{4}{|c|}{ Recent } & & \\
\hline & \multicolumn{2}{|c|}{ Positive } & \multicolumn{2}{|c|}{ Negative } & \multicolumn{2}{|c|}{ Positive } & \multicolumn{2}{|c|}{ Negative } & \multicolumn{2}{|c|}{ Positive } & \multicolumn{2}{|c|}{ Negative } & & \\
\hline & $M$ & $S D$ & $M$ & $S D$ & $M$ & $S D$ & $M$ & $S D$ & $M$ & $S D$ & $M$ & $S D$ & $M$ & $S D$ \\
\hline $\begin{array}{l}\text { Young } \\
(n=32)\end{array}$ & 973 & 204 & 1,031 & 203 & 1,009 & 221 & 1,027 & 202 & 1,001 & 228 & 1,114 & 242 & 87 & 86 \\
\hline $\begin{array}{l}\text { Elderly } \\
(n=6)\end{array}$ & 1,212 & 155 & 1,347 & 185 & 1,331 & 299 & 1,374 & 190 & 1,260 & 279 & 1,464 & 215 & 90 & 149 \\
\hline $\begin{array}{l}\text { Frontal } \\
\quad(n=6) \\
\text { R.C. controls }\end{array}$ & 1,443 & 290 & 1,463 & 142 & 1,446 & 228 & 1,406 & 117 & 1,402 & 203 & 1,499 & 70 & 94 & 114 \\
\hline $\begin{array}{l}(n=8) \\
\text { R.C. }\end{array}$ & $\begin{array}{r}995 \\
1,374\end{array}$ & 163 & $\begin{array}{l}1,100 \\
1,604\end{array}$ & 255 & $\begin{array}{l}1,037 \\
1,405\end{array}$ & 174 & $\begin{array}{l}1,009 \\
1,658\end{array}$ & 126 & $\begin{array}{l}1,048 \\
1,494\end{array}$ & 147 & $\begin{array}{l}1,172 \\
2,325\end{array}$ & 194 & $\begin{array}{l}163 \\
667\end{array}$ & 116 \\
\hline
\end{tabular}

Note-The interference effect is the difference between the recent-negative and the nonrecent-negative trials. 
Table 3

Mean Error Rates (Percentages; With Standard Deviations) for All Subject Groups for Six Trial Types of the Item Recognition Task Trial Type

\begin{tabular}{|c|c|c|c|c|c|c|c|c|c|c|c|c|c|c|}
\hline \multirow{3}{*}{$\begin{array}{l}\text { Subject } \\
\text { Group }\end{array}$} & \multicolumn{4}{|c|}{ Control } & \multicolumn{4}{|c|}{ Nonrecent } & \multicolumn{4}{|c|}{ Recent } & \multirow{2}{*}{\multicolumn{2}{|c|}{$\begin{array}{l}\text { Interference } \\
\text { Effect }\end{array}$}} \\
\hline & \multicolumn{2}{|c|}{ Positive } & \multicolumn{2}{|c|}{ Negative } & \multicolumn{2}{|c|}{ Positive } & \multicolumn{2}{|c|}{ Negative } & \multicolumn{2}{|c|}{ Positive } & \multicolumn{2}{|c|}{ Negative } & & \\
\hline & $M$ & $S D$ & $M$ & $S D$ & $M$ & $S D$ & $M$ & $S D$ & $M$ & $S D$ & $M$ & $S D$ & $M$ & $S D$ \\
\hline $\begin{array}{l}\text { Young } \\
(n=32)\end{array}$ & 3.8 & 5.8 & 0.5 & 1.5 & 2.7 & 4.4 & 0.5 & 1.4 & 1.6 & 2.2 & 1.3 & 2.0 & 0.8 & 2.2 \\
\hline $\begin{array}{c}(n=6) \\
\text { Frontal }\end{array}$ & 6.2 & 10.7 & 5.8 & 9.7 & 10.5 & 15.3 & 3.8 & 6.6 & 9.6 & 8.6 & 11.3 & 10.2 & 7.5 & 4.3 \\
\hline $\begin{array}{l}\text { Frontal } \\
(n=6) \\
\text { R.C. controls }\end{array}$ & 28.1 & 18.1 & 6.7 & 9.8 & 28.3 & 14.6 & 7.4 & 7.4 & 27.1 & 16.9 & 12.1 & 9.3 & 4.7 & 7.8 \\
\hline $\begin{array}{l}\quad(n=8) \\
\text { R.C. }\end{array}$ & $\begin{array}{l}11.8 \\
21.1\end{array}$ & 15.3 & $\begin{array}{r}3.8 \\
10.0\end{array}$ & 6.9 & $\begin{array}{r}9.4 \\
35.0\end{array}$ & 11.8 & $\begin{array}{r}1.0 \\
11.1\end{array}$ & 2.1 & $\begin{array}{r}5.0 \\
12.5\end{array}$ & 10.3 & $\begin{array}{r}5.0 \\
30.0\end{array}$ & 5.0 & $\begin{array}{r}4.0 \\
18.9\end{array}$ & 4.4 \\
\hline
\end{tabular}

Note-The interference effect is the difference between the recent-negative and the nonrecent-negative trials.

each group are given in Tables 2 and 3. The first two trials in each block (with the exception of the control block) were excluded, since they could not be assigned to either the recent or the nonrecent conditions, using our two-trial history criteria. Comparisons between subject groups were made to assess two effects: (1) the effect of aging and frontal damage on baseline working memory performance and (2) the effect of aging and frontal damage on interference effects in working memory. In order to assess baseline working memory performance, comparisons were made between subject groups on both the nonrecent trials and the control trials. In order to assess interference effects, the critical comparison was the difference in performance between the recent-negative and the nonrecent-negative trials. The magnitude of this interference effect was compared between young and elderly subjects, between elderly control subjects and frontal patients, and between frontal patients and patient R.C.

\section{Working Memory Performance}

In the initial analysis of working memory performance across subject groups, we combined the data of the elderly control group and the R.C.-control group into one group (age-matched control subjects), and we combined the data of the frontal patients and patient R.C. into another group (frontal; see Figure 4). A one-way analysis of variance (ANOVA) of response latencies from the nonrecent trials from young, elderly, and frontal subjects revealed a significant main effect of subject group $[F(2,50)=12.24$, $p<.01]$. Planned contrasts indicated that young subjects $(M=1,018 \mathrm{msec})$ responded more quickly than did agematched subjects $(M=1,164 \mathrm{msec})$ and frontal $(M=$ $1,441 \mathrm{msec})$ subjects $(t=4.66, p<.01)$, and that agematched subjects responded more quickly than did frontal subjects $(t=2.85, p<.01)$. Likewise, an ANOVA of error rates from young, age-matched, and frontal subjects revealed a significant main effect of subject group $[F(2,50)=$ $25.89, p<.01]$. Planned contrasts indicated that young subjects $(M=1.8 \%)$ made fewer errors than did agematched $(M=6.2 \%)$ and frontal $(M=18.8 \%)$ subjects $(t=$ $6.45, p<.01)$ and that age-matched subjects made fewer errors than did frontal subjects $(t=4.78, p<.01)$.
These patterns were replicated in the control task: There was a significant main effect of subject group on both response latency $[F(2,50)=16.45, p<.01]$ and error rate $[F(2,50)=15.38, p<.01]$. A between-subjects comparison of the control trials and the nonrecent trials indicated no difference in response latency or error rate $(t \mathrm{~s}<1)$.

In order to examine the working memory performance of patients with frontal lesions in more detail, a singlecase approach was used in which we tested whether the performance of a given individual differed significantly from that of the control group. Because it may be incorrect to treat the diverse frontal patients as a homogeneous group, this approach allowed us to identify any patients who were performing outside of the normal range on this task. Using the control trials as a baseline measure of working memory, the performance of each patient was compared with the mean and standard deviation of the age-matched group. Any patient who scored more than 1.64 standard deviations beyond the mean (approximately the 95 th percentile) was considered impaired. The performance for each patient is reported in Table 4. Three patients (J.M., W.A., and R.C.) had response times that exceeded the cutoff for normal response time $(1,483 \mathrm{msec})$. Likewise, 3 patients (M.F., J.M., and W.A.) had error rates that exceeded the cutoff for normal performance $(21 \%)$.

\section{Interference Effects}

In young control subjects, we observed a significant interference effect on response time $[t(31)=5.74, p<.01]$. Young subjects were, on average, $87 \mathrm{msec}(8.5 \%)$ slower to respond no to a recent-negative trial than to a nonrecentnegative trial. The magnitude of this effect was comparable to that reported in previous studies (e.g., D'Esposito et al., 1999). There was a trend for young subjects to make more errors ( $1 \%$ difference) on recent-negative trials than on nonrecent-negative trials $[t(31)=2.03, p<.06]$.

As in the above analyses of working memory performance, we initially analyzed the interference effect (recentnegative - nonrecent-negative) by comparing young, age-matched, and frontal subject groups with a one-way ANOVA, with group as the between-subjects factor. The analysis of response latencies revealed no effect of group 

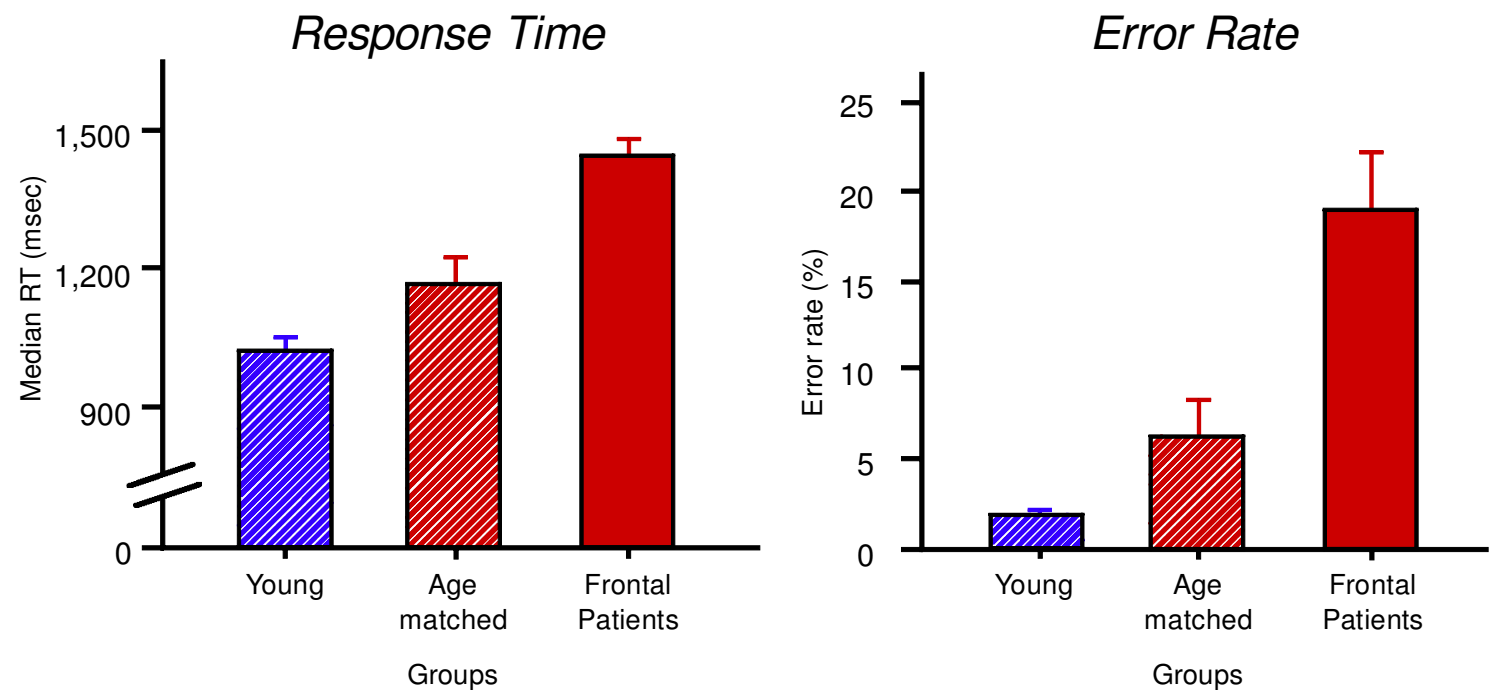

Figure 4. Baseline working memory performance (response times and error rates) on the nonrecent (positive and negative) trials for young subjects $(n=32)$, age-matched control subjects $(n=14)$, and patients with frontal lesions $(n=7)$. One standard error of the mean is indicated above each bar.

$(F=1.68$, n.s. $)$. However, the analysis of error rates revealed a main effect of group $(F=8.59, p<.01)$. Planned contrasts indicated that young subjects $(M=1 \%)$ showed a smaller interference effect than did age-matched $(M=$ $5 \%)$ and frontal $(M=7 \%)$ subjects $(t=4.10, p<.01)$ but that age-matched subjects did not differ from frontal subjects $(t<1)$. It should be noted that young subjects were performing near ceiling, so a smaller interference effect in error rates of young subjects is difficult to interpret. One way to address this ceiling effect is to select a subset of young and age-matched subjects that are matched in overall error rate and then to compare the interference effects in these subjects. The median error rates for the young and the age-matched subjects were used to select the young subjects who had a relatively high error rate $(n=20, M=$ $3 \%$ ) and the age-matched subjects who had a relatively low error rate $(n=12, M=3 \%)$. These subsets of subjects were matched in terms of their overall error rate for nonrecent trials $[t(30)=0.27]$, as well as in their error rates for negative-nonrecent trials $[t(30)=0.34]$. Even in these
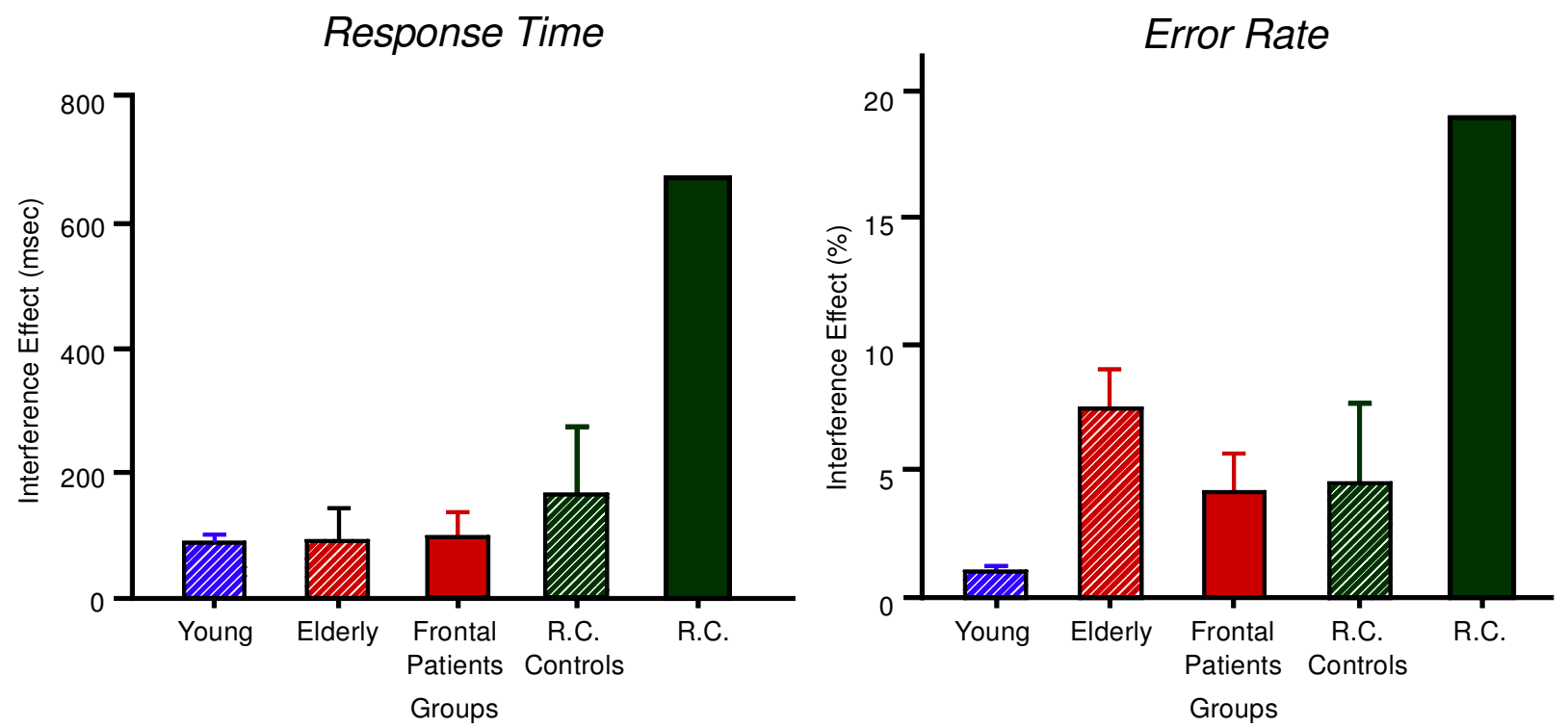

Figure 5. Interference effects on response times (RTs) and error rates (recent-negative trials - non recent-negative trials) for young $(n=32)$, elderly $(n=6)$, frontal $(n=6)$, R.C. age-matched control subjects $(n=8)$, and R.C. For young, elderly, and frontal groups, one standard error of the mean is indicated above each bar. For the R.C. age-matched control subjects, one standard deviation is indicated above the bar, to allow for comparison of that group with a single individual. 
matched samples, young subjects showed a smaller interference effect than did the age-matched controls $[t(30)=$ $4.02, p<.01]$. Thus, it appears unlikely that this difference between young and age-matched subjects can be explained entirely as a scaling artifact.

Although there were no group differences between agematched and frontal subjects in the magnitude of the interference effect (in either response latency or error rate), we examined the performance of R.C. separately from the rest of the patients with frontal lesions, because R.C. was the one patient whose lesion impinged on the area of the prefrontal cortex implicated in previous neuroimaging studies of this effect. As was described above, we compared each patient's performance with the mean and standard deviation of the interference effect in the control group. In light of hypothesized differences between R.C. and the other frontal patients, these comparisons were made with the relevant control group (see Figure 5). That is, patient R.C. was compared with the control group matched to his (younger) age and education, and the remaining frontal patients were compared with their control group. Patient R.C.'s interference effect in response latency $(667 \mathrm{msec})$ and error rate (19\%) exceeded the cutoffs (354 msec and $11 \%$ ) for normal performance in his control group. In fact, R.C.'s interference effect was more than four standard deviations above the mean of his control group for response time and more than three standard deviations above the mean for error rate. For the remaining frontal patients, none exceeded the cutoffs (334 msec and 14\%) for normal performance in their control group (see Table 4).

In order to test whether R.C. differed from the remaining frontal patients, a similar analysis approach was employed: The performance of R.C. was compared with the mean and standard deviation of the other 6 frontal patients. In terms of baseline working memory performance (nonrecent trials), R.C.'s performance was within the range of the other frontal patients; both his response times and error rates were within one standard deviation of the group mean. However, the interference effect exhibited by R.C. fell outside the range of performance for the other frontal patients. His interference effect on response time $(667 \mathrm{msec})$ was more than five standard deviations above the mean of the frontal patients, and his interference effect on error rate $(19 \%)$ was nearly two standard deviations above their mean.

One possible explanation of the seemingly exaggerated interference effect exhibited by R.C. is that the more impaired a patient's working memory is, the larger the interference effect will be. In order to examine the relationship between working memory performance and the magnitude of the interference effect, we calculated the correlation between our measure of baseline working memory performance (i.e., control trials) and the magnitude of the interference effect (i.e., recent-negative - nonrecentnegative). Across all of our patients and their age-matched control subjects, the correlation was quite low (.18 for error rate and -.02 for response time, $p s>.40$ ). As can be seen in Figure 6, there was no relationship in any of the elderly or frontal groups between baseline working memory performance and interference effect. In this figure, it is apparent that R.C.'s baseline working memory performance was within the range of performance of the other frontal patients, but his interference effect was an outlier for both response time and error rate. Note that several other patients with poorer baseline working memory performance exhibited much smaller interference effects.

\section{DISCUSSION}

In this study, we examined working memory performance on an item recognition test and, specifically, interference effects in working memory in a group of patients with unilateral frontal lesions. In particular, we examined the performance of 1 patient (R.C.) who uniquely had a lesion that included the region of the prefrontal cortex (Brodmann's area 45) identified in neuroimaging studies of interference effects in working memory (D'Esposito et al., 1999; Jonides et al., 1998). In comparison with age-matched control subjects, we found a slight, but reliable, working memory impairment in patients with frontal lobe lesions. However, only patient R.C. showed an exaggerated interference effect: On trials in which the probe was not a member of the current target set but was a member of the prior target set (i.e., recent-negative trials), R.C. was nearly $50 \%$ slower and made almost three times as many errors than on nonrecent-negative trials. This marked interfer-

Table 4

For Each Patient, Age at Testing, Lesion Volume, Baseline Working Memory Performance (Control Trials), and Interference Effect (Recent-Negative - Nonrecent-Negative)

\begin{tabular}{|c|c|c|c|c|c|c|}
\hline \multirow[b]{2}{*}{ Patient } & \multirow[b]{2}{*}{ Age } & \multirow{2}{*}{$\begin{array}{c}\text { Lesion } \\
\text { Volume }(\mathrm{cc})\end{array}$} & \multicolumn{2}{|c|}{ Baseline Working Memory } & \multicolumn{2}{|c|}{ Interference Effect } \\
\hline & & & Errors $(\%)$ & $\mathrm{RT}(\mathrm{msec})$ & Errors $(\%)$ & $\mathrm{RT}(\mathrm{msec})$ \\
\hline W.E. & 69 & 41 & 11 & 1,427 & 2 & 60 \\
\hline M.F. & 65 & 40 & $24 *$ & 1,370 & 7 & 0 \\
\hline E.B. & 81 & 17 & 5 & 1,328 & -1 & 203 \\
\hline J.M. & 56 & 19 & $36^{*}$ & $1,728 *$ & -4 & 221 \\
\hline W.A. & 77 & 26 & $26^{*}$ & $1,634 *$ & 13 & -64 \\
\hline N.T. & 58 & 20 & 3 & 1,233 & 13 & 141 \\
\hline R.C. & 51 & 53 & 16 & $1,489 *$ & $19 *$ & $667^{*}$ \\
\hline
\end{tabular}

*Scores that exceeded the cutoff for normal performance (1.64 standard deviations above the mean for age-matched control subjects). 
Response Time

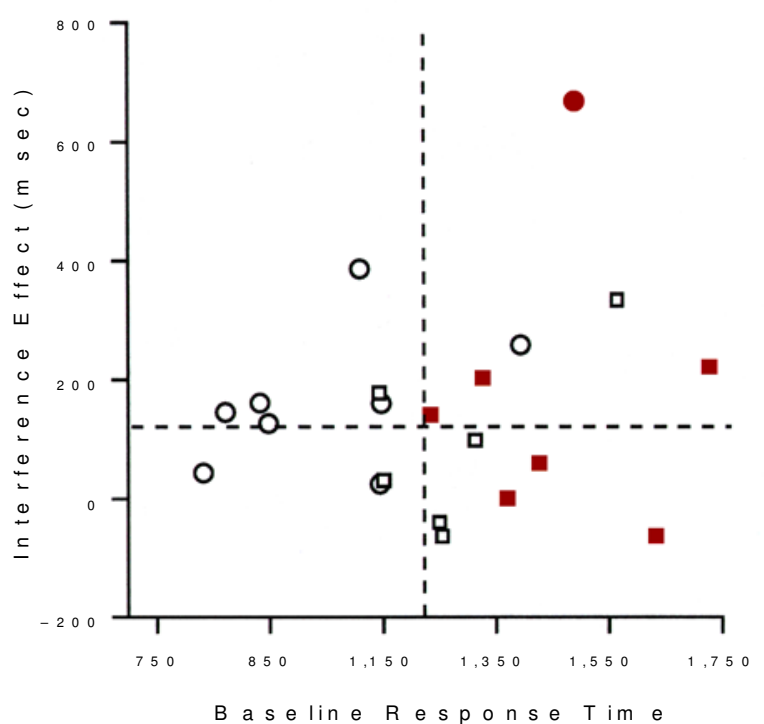

Error Rate

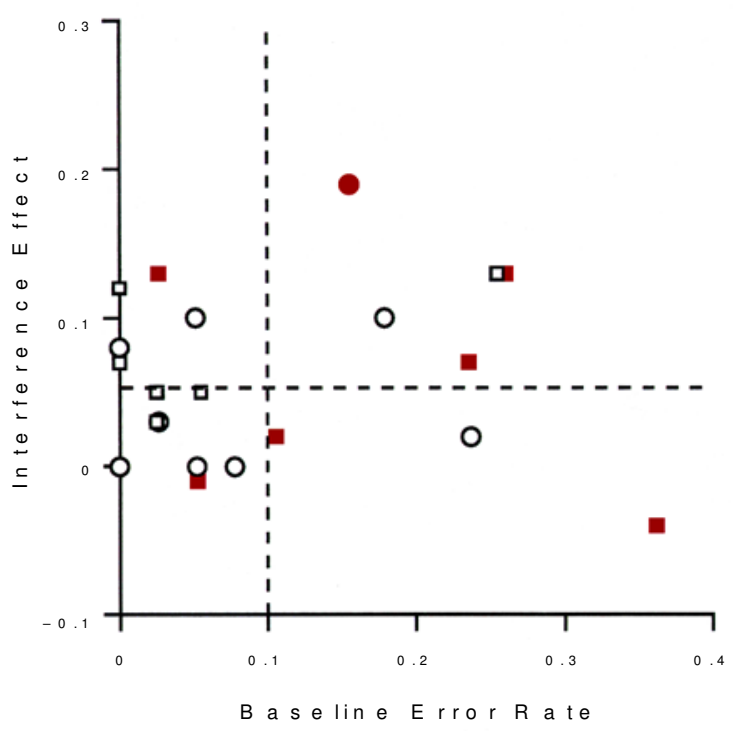

口Elderlycontrols O R.c.controls
Erontalpatients

Figure 6. Scatterplots illustrating the relation between baseline working memory performance (control trials) and the interference effect (recent-negative trials - nonrecent-negative trials) in frontal patients and age-matched control subjects. Patients are indicated by filled symbols; control subjects are indicated by open symbols. Patient R.C. and his control subjects are indicated by circles; the other frontal patients and their control subjects are indicated by squares. The dashed lines indicate the mean for each variable. As is evident in the plots of both response times and error rates, R.C.'s baseline performance was comparable to that of other frontal patients, but his interference effect was markedly exaggerated. Note that no relationship between baseline working memory performance and interference effect was observed (both $r \mathrm{~s}<.20)$.

ence effect distinguished him from 6 other frontal patients, who performed comparably to R.C. for all trial types except for the recent-negative trials. That is, R.C. did not have a more significant working memory impairment overall; rather, he differed from the other frontal patients only with regard to the magnitude of his interference effect. On the basis of this pattern of performance, we propose that there are at least two dissociable components of working memory involved in this item recognition task: a mnemonic component, such as the phonological loop first described by Baddeley (1986), and a nonmnemonic component that prevents catastrophic interference effects, of the sort observed with R.C. We can examine changes in these two putative components in each of our subject groups.

\section{Effects of Normal Aging}

In the comparison of young (mean age $=19$ years) with older (mean age $=56$ years) subjects, we observed evidence for age-related changes in both mnemonic and nonmnemonic components of working memory. Older subjects were slower, made more errors on the baseline working memory items, and exhibited a more pronounced effect of interference on error rate (but not on response time). This finding replicated the results of a recent study (Jonides et al., 2000) in which elderly performance and PET activation were examined with the same modified item recog- nition paradigm as that used in the present study. In addition to the behavioral effects, elderly subjects in Jonides et al.'s study showed less PET activation in the left inferior frontal region that was associated with the interference effect in young subjects.

Age-related declines in working memory have been well documented, although the mechanisms underlying these changes have not been established. Baddley (1986) initially proposed a decrease in the capacity of the central executive component of working memory. More recently, evidence has supported the claim that central executive functions, rather than storage capacity, are affected in aging (Van der Linden, Bredart, \& Beerten, 1994). However, other studies have concluded that there are age-related declines in the capacity of the phonological loop (Meguro et al., 2000). Some investigators have argued that the capacities of both the phonological loop and the central executive are preserved with aging but that a general slowdown in perceptual speed explains the changes in performance (Fisk \& Warr, 1996; Salthouse, 1985). Finally, the authors of a recent ERP study concluded that there are multiple distinct effects of aging on working memory (Pelosi \& Blumhardt, 1999). Although the present study was not designed to explicitly test these competing hypotheses, our results are consistent with accounts of multiple sources of working memory impairments in aging. 


\section{Effects of Frontal Lobe Lesions}

In the comparison of patients having frontal lesions with age-matched control subjects, we observed impairments in baseline working memory performance, but no changes in the magnitude of the interference effect. This is clearly illustrated in the scatterplots in Figure 6, which show a shift in the distribution of baseline performance of the frontal patients (indicated by filled symbols), as compared with the control subjects (indicated by open symbols), but no change in the distribution of the interference effect. This result appears to contradict the meta-analysis of the effects of frontal lesions on working memory performance described earlier (D'Esposito \& Postle, 1999). The conclusions of that analysis were that frontal lesions were more likely to affect working memory tasks that require attentional or inhibitory mechanisms (e.g., distracting stimuli during the delay) that are not required in a simple delayed response task. The apparent discrepancy between the conclusions of their meta-analysis and those of the present study (which would be classified as a simple delayed response task) could reflect relevant differences in either the patient samples or the task.

One possible explanation for the discrepancy is that the meta-analysis was based on a diverse collection of frontal patients; when patients with heterogeneous lesions are grouped together, certain patterns of deficits can be obscured. Perhaps deficits on the item recognition task are evident only in patients with certain locations of frontal damage. A second possible explanation is that the working memory impairment we observed may be specific to our stimulus set (i.e., letters). None of the studies reviewed by D'Esposito and Postle (1999) used item recognition tasks with letters; rather, they used either spatial tasks (e.g., visual point localization) or nonspatial tasks with nonverbal stimuli (e.g., memory for forms, nonsense figures, sounds, and colors). We also varied the visual form of the stimulus (i.e., we used lowercase letters in the target set and uppercase letters as a probe) to encourage more verbal or symbolic processing; this may have resulted in differences between our study and those reviewed in the meta-analysis.

A third possible difference between previous delayed response tasks and the task used in the present study was a feature of the design we used: Each consecutive pair of target sets had $50 \%$ overlap between items. For example, if one trial had the letters " $b, r, g$, a" to remember, the next trial might have the letters " $k, b, s$, g." This was done to ensure that repetition of target letters was not confounded with the trial type (e.g., the recent-positive trials require such repetition). However, one could argue that the repetition of items across trials increased the attentional requirements of the task, thus making it more sensitive to frontal lobe lesions. There are two reasons to think this is not the case. First, if that were the explanation of the impairment in these patients, one also would have expected them to show a pronounced interference effect, which they did not. Second, we included the block of control trials to address this potential concern; the control trials were constructed without any repetition of items on consecutive trials. There were no differences in performance between the control trials and the nonrecent trials of the item recognition task with repeating stimuli.

\section{Effects of Inferior Frontal Lesions}

One patient in our sample, R.C., was identified because his lesion included the region of the inferior frontal gyrus (Brodmann's area 45) identified by previous neuroimaging studies of the interference effect in working memory (D'Esposito et al., 1999; Jonides et al., 1998). Although R.C.'s lesion was large and was certainly not confined to the region identified in those studies, he was the only patient in our sample to have a lesion that included the more anterior aspects of the inferior frontal gyrus. This area can be seen in Slices 3-6 of his lesion reconstruction in Figure 2. In comparison with the other frontal patients (and in comparison with his age-matched control subjects), patient R.C. showed a significantly exaggerated interference effect. His baseline working memory performance was in the range of the other frontal patients; that is, his working memory was impaired but not atypical of the other patients with frontal lesions. However, the magnitude of his interference effect was clearly atypical. R.C. made three times as many errors on recent-negative trials as he did on nonrecent-negative trials, and he was 50\% slower for those recent-negative trials that he did answer correctly than for the nonrecent trials. His 667-msec interference effect on response time was more than five standard deviations above the mean for the other frontal patients. ${ }^{1}$

In an attempt to understand why R.C. showed an exaggerated interference effect, we have focused on the unique location of his lesion, in that it involves the anterior aspects of the inferior frontal gyrus. However, as is to be expected with lesion studies in humans, there are likely to have been other differences between R.C. and the other frontal patients that should be considered. First, R.C. had the largest lesion in the sample. However, we do not believe that this factor alone can account for his impairment. As is shown in Table 4, 2 control patients (W.E. and M.F.) with large lesions showed normal interference effects; the control patient with the largest interference effect (N.T.) had one of the smallest lesions. In fact, the correlation between lesion size and interference effect was not significant. Second, the etiology of R.C.'s lesion was different from the etiology of any of the other patients. R.C.'s lesion was the result of surgery following a ruptured arteriovenous malformation, whereas the other patients had lesions resulting from nonhemorrhagic infarction. Comparisons between hemorrhagic and nonhemorrhagic etiologies are particularly problematic when imaging data are available only from the acute period. However, in the present study, we based our reconstructions on chronic lesion information that documented clear differences in the regions of prefrontal damage between R.C. and the other patients.

\section{Implications for Theories of Working Memory}

On the basis of the patterns of deficits described above, we propose that both mnemonic and nonmnemonic processes contribute to performance on the item recognition test of working memory and that these two processes can 
be dissociated from one another. Specifically, our results suggest that broad regions of the prefrontal cortex contribute to the mnemonic processes involved in working memory but that the left inferior frontal gyrus has a more specialized role in the nonmnemonic processes. Our evidence for this can be summarized with these three major findings: (1) Patients with lesions to a variety of prefrontal regions (dorsal and ventral, left and right) exhibited an impairment in working memory but normal interference effects, as compared with control subjects; (2) R.C., who uniquely had a lesion to the anterior extent of the inferior frontal gyrus, showed a marked interference effect, but his baseline working memory performance was comparable to that of the other frontal patients; (3) across all patients and their age-matched control subjects, there was no relation between baseline working memory performance and the magnitude of the interference effect, either in response time or accuracy, further supporting the claim that these are independent processes.

For purposes of simplification and contrast, we have described the interference effect as the reflection of one component of working memory, a nonmnemonic process, and we have described the baseline working memory performance as the result of a second, mnemonic component. The latter statement is clearly an oversimplification. There are likely multiple processes involved even in the baseline working memory trials. For example, using a similar item recognition task and also a two-back working memory task, Awh et al. (1996) provided evidence for a dissociation between storage and rehearsal processes. Their PET experiment identified regions of the prefrontal cortex that were involved in rehearsal and regions of the posterior parietal cortex involved in storage. In the present investigation, we did not attempt to distinguish between these different components. Rather, we focused on the goal of contrasting that set of components with a specific nonmnemonic component that results in the interference effect.

What is the process underlying this interference effect, the process that has been altered by R.C.'s lesion? One possibility is that it is a processing component specific to working memory and, perhaps, makes up part or all of the central executive. Many studies have found support for the claim that the central executive is mediated by the prefrontal cortex. For example, Postle, Berger, and D'Esposito (1999) described a double dissociation between working memory executive control processes subserved by the prefrontal cortex and working memory storage processes subserved by the posterior parietal cortex. However, the region of the prefrontal cortex that is often linked to the central executive is considerably superior to the location of R.C.'s lesion and the locus of activity of the interference effect. A second possibility is that the process is a more general mechanism that is engaged by many tasks, including, but not limited to, working memory. For example, we have previously described the role of the left inferior frontal gyrus in the selection of information from among competing alternatives in semantic memory ${ }^{2}$ (ThompsonSchill, D'Esposito, Aguirre, \& Farah, 1997; ThompsonSchill et al., 1998). Activity in that region is sensitive to manipulations of recent experience (i.e., priming) that increase the availability of competing or irrelevant information (Thompson-Schill, D'Esposito, \& Kan, 1999). The interference effect under investigation in the present study could be described in similar terms: A recently presented probe item will have higher activation than a new probe item, which will affect the amount of competition against the items in the current target set.

There are two other possibilities that might be considered for R.C.'s exaggerated interference effect. First, one might suppose that we are simply observing an effect of difficulty, so that performance is worst on the most difficult condition as the result of a generalized performance deficit (Chapman \& Chapman, 1973). Although this issue would be most convincingly addressed by subsequent experimentation, there is some reason to believe this is not the case, based on our present data: If interference increased as a function of a generalized performance deficit (e.g., poor motivation, general inattentiveness), one would expect to observe a systematic relationship between working memory performance and interference. We did not observe such a correlation in this study (see Figure 6). Second, R.C. could have been adopting a familiarity-based strategy (as opposed to working memory encoding or rehearsal) that would have increased errors on recent-negative trials. The issue here is not whether R.C. was influenced by familiarity when making his decision; this was the basis for the increased competition that we have described above. Rather, at issue is whether R.C. made a strategic choice (albeit an ineffective one) to perform the task differently than the other control patients. This is not an alternative we can rule out at present. Recent studies have documented the role of the prefrontal cortex in selection from working memory (Rowe, Toni, Jospephs, Frackowiak, \& Passingham, 2000), using procedures that did not include a familiarity manipulation, which may be useful in addressing this issue in the future.

The frontal lobes have been linked to working memory performance in many studies. Our results and the results of others underscore the importance of careful characterization of the components of working memory and their relation to specific regions of the frontal lobes, emphasizing the multicomponential nature of the working memory system. However, our understanding of working memory and its relation to different brain regions might require reframing some ideas about working memory processes in terms of more general processing mechanisms, rather than delineating specialized parts of a working memory system. In this study, we have focused on a putative selection mechanism, linked to the left inferior frontal gyrus in previous research, that may be required during certain working memory tasks (i.e., our nonrecent-negative trials) but that may also be required outside the domain of working memory (e.g., semantic processing; see Thompson-Schill et al., 1998). Similarly, other processes might also be called upon for certain working memory tasks, such as attention shifting (e.g., Garavan, 1998), coordination of multiple tasks (D'Esposito et al., 1995), and so forth. That is, the search for the elusive central executive of working memory might 
lead us to the conclusion that there is no such specialized system. This approach might shift future research questions in this area from "which parts of working memory are linked to which areas of the frontal lobes?" to "which functions of the frontal lobes are required by which types of working memory problems?"

\section{REFERENCES}

Atkinson, R C., \& Shiffrin, R. M. (1968).Human memory: A proposed system and its control processes. In K. Spence \& J. Spence (Eds.), The psychology of learning and motivation (Vol. 2, pp. 89-195). New York: Academic Press.

Awh, E., Jonides, J., Smith, E. E., Schumacher, E. H., Koeppe, R. \& KATz, S. (1996). Dissociation of storage and rehearsal in verbal working memory: Evidence from PET. Psychological Science, 7, 25-31.

BADDELEY, A. D. (1986). Working memory. Oxford: Oxford University Press.

BALDO, J. V. (1997). Spatial and object working memory in patients with lateral prefrontal cortex lesions. Berkeley: University of California Press.

Basso, A., Spinnler, H., Vallar, G., \& Zanobio, E. (1982). Left hemisphere damage and selective impairment of auditory verbal short-term memory: A case study. Neuropsychologia, 20, 263-274.

Bechara, A., Damasio, H., Tranel, D., \& Anderson, S. W. (1998). Dissociation of working memory from decision making within the human prefrontal cortex. Journal of Neuroscience, 18, 428-437.

Chao, L., \& KNight, R. T. (1998). Contribution of human prefrontal cortex to delay performance. Journal of Cognitive Neuroscience, 10, 167177.

Chapman, L. J., \& Chapman, J. P. (1973). Problems in the measurement of cognitive deficits. Psychological Bulletin, 79, 380-385.

D'Esposito, M., Detre, J. A., Alsop, D. C., Shin, R. K., Atlas, S., \& Grossman, M. (1995). The neural basis of the central executive system of working memory. Nature, 378, 279-281.

D'Esposito, M., \& Postle, B. R. (1999). The dependence of span and delayed-response performance on prefrontal cortex. Neuropsychologia, 37, 1303-1315.

D’Esposito, M., Postle, B. R., Jonides, J., \& Smith, E. E. (1999). The neural substrate and temporal dynamics of interference effects in working memory as revealed by event-related functional MRI. Proceedings of the National Academy of Sciences, 96, 7514-7519.

FISK, J. E., \& WARR, P. (1996). Age and working memory: The role of perceptual speed, the central executive, and the phonological loop. Psychology \& Aging, 11, 316-323.

Fuster, J. M., \& AleXander, G. E. (1971). Neuron activity related to short-term memory. Science, 173, 652-654.

Garavan, H. (1998). Serial attention within working memory. Memory \& Cognition, 26, 263-276.

Ghent, L., Mishrin, M., \& Teuber, H. L. (1962). Short-term memory after frontal-lobe injury in man. Journal of Comparative \& Physiological Psychology, 5, 705-709.

Hanley, J. R., Young, A. W., \& Pearson, N. A. (1991). Impairment of the visuospatial sketch pad. Quarterly Journal of Experimental Psychology, 43A, 101-125.

JACOBSEN, C. F. (1935). Functions of frontal association areas in primates. Archives of Neurology \& Psychiatry, 33, 558-560.

Jonides, J., Marshuetz, C., Smith, E. E., Reuter-Lorenz, P. A., Koepre, R. A., \& Hartley, A. (2000). Age differences in behavior and PET activation reveal differences in interference resolution in verbal working memory. Journal of Cognitive Neuroscience, 12, 188196.

Jonides, J., Smith, E. E., Marshuetz, C., Koeppe, R. A., \& ReuterLoRENZ, P. A. (1998). Inhibition in verbal working memory revealed by brain activation. Proceedings of the NationalAcademy of Sciences, 95, 8410-8413.

Meguro, Y., Fujit, T., Yamadori, A., Tsukiura, T., Suzuki, K. OKUDA, J., \& OSAKA, M. (2000). The nature of age-related decline on the reading span task. Journal of Clinical \& Experimental Neuropsychology, 22, 391-398.
Pelosi, L., \& Blumhardt, L. D. (1999). Effects of age on working memory: An event-related potential study. Cognitive Brain Research, 7, 321-334.

Post Le, B. R., Berger, J. S., \& D'Esposito, M. (1999). Functionalneuroanatomical double dissociation of mnemonic and executive control processes contributing to working memory performance. Proceedings of the National Academy of Sciences, 96, 12959-12964.

Ptito, A., Crane, J., Leonard, G., Amsel, R., \& Caramanos, Z. (1995). Visual-spatial localization by patients with frontal-lobe lesions invading or sparing area 46. NeuroReport, 6, 1781-1784.

Rowe, J., Toni, I., Jospephs, O., Frackowiak, R., \& Passingham, R. (2000). The prefrontal cortex: Response selection or maintenance within working memory? Science, 288, 1556-1560.

Salthouse, T. A. (1985). Speed of behavior and its implications for cognition. In J. E. Birren \& K. W. Schaie (Eds.), Handbook of the psychology of aging (pp. 400-426). New York: Van Nostrand-Reinhold.

Salthouse, T. A. (1992). Mechanisms of age-cognition relations in adulthood. Hillsdale, NJ: Erlbaum.

Thompson-Schill, S. L., D’Esposito, M., Aguirre, G. K., \& FARAH, M. J. (1997). Role of eft inferior prefrontal cortex in retrieval of semantic knowledge: A reevaluation. Proceedings of the National Academy of Sciences, 94, 14792-14797.

Thompson-Schill, S. L., D’Esposito, M., \& Kan, I. P. (1999). Effects of repetition and competition on prefrontal activity during word generation. Neuron, 23, 513-522.

Thompson-Schill, S. L., Swick, D., Farah, M. J., D'Esposito, M., KAN, I. P., \& KNIGHT, R. T. (1998). Verb generation in patients with focal frontal lesions: A neuropsychological test of neuroimaging findings. Proceedings of the National Academy of Sciences, 95, 15855 15860 .

Van der Linden, M., Bredart, S., \& Beerten, A. (1994). Age-related differences in updating working memory. British Journal of Psychology, 85, 145-152.

Wilson, F. A. W., Scalaidhe, S. P. O., \& Goldman-Rakic, P. S. (1993). Dissociation of object and spatial processing domains in primate prefrontal cortex. Science, 260, 1955-1958.

\section{NOTES}

1. Although the focus of the present study was on the interference effect in recent-negative trials, one could also examine whether a facilitation effect was present in recent-positive trials, as compared with nonrecentpositive trials. Such a facilitation effect has not been reliably observed in young subjects, either in previous studies using this task or in the present study. As can been seen in Tables 2 and 3, R.C. showed what might be a large facilitation effect in error rate; however, in response latency, the effect was in the opposite direction. This discrepancy does not allow us to reject the possibility that R.C. was trading speed for accuracy. Following Salthouse (1992), we computed a composite measure of facilitation effect size on response latency and error rate, using normalized $z$ scores. With this composite measure, there were no differences between any subject groups. (R.C.'s normalized facilitation effect size was about $1 S D$ above the mean for his control group, in contrast to his normalized interference effect size, which was greater than $4 S D$ s above the mean for his control group.)

2 . The region of the inferior frontal gyrus identified by ThompsonSchill et al. (1997; Thompson-Schill et al., 1999) and the region identified by Jonides et al. (1998) and D'Esposito et al. (1999) may be adjacent but distinct. The local maxima of activity in the studies of selection of semantic information were centered in Brodmann's area 44. The local maxima of activity in the studies of interference effects on working memory were centered just anteriorly, in Broadmann's area 45. Although the extent of activation in the inferior frontal gyrus in these studies was somewhat overlapping, it is possible that within the inferior frontal regions, there are distinct domain-specific specializations that are reflected in these subtle differences in the local maxima of activation.

(Manuscript received July 9, 2001; revision accepted for publication April 17, 2002.) 